Editorial

\title{
Syphilis in Pregnancy and Congenital Syphilis: Why Can We not yet Face This Problem?
}

\section{Sífilis na gestação e sífilis congênita: por que ainda não conseguimos enfrentar esse problema?}

\author{
Helaine Milanez ${ }^{1}$ \\ ${ }^{1}$ Department of Obstetrics and Gynecology, Faculdade de Ciências \\ Médicas, Universidade Estadual de Campinas - Unicamp, Campinas, \\ SP, Brazil
}

Rev Bras Ginecol Obstet 2016;38:425-427.

The increased occurrence of syphilis during pregnancy, and its severe repercussions to the fetal environment, has been a cause for concern among health care professionals. It is hard to comprehend why congenital syphilis has not yet been controlled, since its agent, the spirochaete Treponema pallidum, is well known and is highly susceptible to penicillin, a low cost and easily available drug. When we compare the control of mother-to-child transmission (MTCT) of HIV with that of syphilis, it is even harder to understand why we have such success with the first one and disastrous results with the second! ${ }^{1}$

When we evaluate the epidemiology of syphilis in pregnancy and in the general adult population, we notice that there were great expectations to eradicate this disease after WWII, when penicillin was discovered, and its high efficacy in controlling Treponema confirmed. There was actually a reduction in the number of cases in the mid-1950s. Nevertheless, there was a significant increase starting in the 1960s, due probably to sexual liberation and the growing use of illicit drugs. A new reduction in the number of cases occurred again in the 1970 s, due to public health campaigns. In the 1980 s, a new growth in the number of cases was detected, strongly associated with the HIV epidemic around the world, sexual promiscuity and the sexual practices of men who have sex with men (MSM). At that time, increasing rates were also observed in heterosexual individuals who were associated with promiscuity, unprotected sexual practices and prostitution. Until today, despite many initiatives to reduce the number of cases, the infection rate in adults is alarming, particularly among pregnant women and newborns. ${ }^{2,3}$

Many public health campaigns launched in Brazil in the last decades, specifically targeting pregnant women, tried to control the disease and prevent the infection in newborns. By the end of the 1980s, a new campaign was launched to "eradicate congenital syphilis by the year 2000". This campaign focused on educating pregnant women about the importance of being tested and treated during prenatal care. Unfortunately, we arrived to the year 2000 with a higher occurrence of congenital syphilis. At this point, a new campaign was created trying to control congenital syphilis by 2012, since eradicating the disease seemed unrealistic. Again, the campaign failed to reach its goals and a higher number of infections was observed. ${ }^{3-5}$

Why is it so hard to face the congenital syphilis problem in our country?

Because syphilis can be easily diagnosed and efficient treatment is available at low cost, potential barriers for the control of the disease have been studied. The role of health care workers is one of them. The new campaign from the Ministry of Health in Brazil is focusing on evaluating the health professional's role in caring for pregnant women and preventing congenital syphilis in newborns. ${ }^{4,5}$

Recent data on syphilis during pregnancy and congenital syphilis in our country are alarming. A significant proportion of women and newborns are infected. Among pregnant women, there was an increase of $1047 \%$ between 2005 and 2013. During this same period, an increase of $135 \%$ in congenital syphilis notifications was observed. These numbers might mean that the notification has improved, which in fact has happened. Nevertheless, this is not enough to explain the epidemic number of cases in pregnant women and newborns. ${ }^{5}$

Data about syphilis cases around the world show that more than 12 million cases a year, registered by the World
Address for correspondence Helaine Milanez, PhD, Department of Obstetrics and Gynecology, Unicamp, R. Tessália Vieira de Camargo, 126. Cidade Universitária Zeferino Vaz, Campinas, SP, 13083-887, Brazil

(e-mail: helaine@caism.unicamp.br).
DOI http://dx.doi.org/ 10.1055/s-0036-1593603. ISSN 0100-7203.
Copyright $\odot 2016$ by Thieme Publicações License terms Ltda, Rio de Janeiro, Brazil 
Health Organization (WHO), happen in the countries with less financial and social resources. They were associated with 90,000 neonatal deaths and to 65,000 premature or low birth weight newborns. The global rates for adverse events varied from 58 to more than $80 \% .{ }^{6}$ It is estimated that, in 2008 , there were $1,360,485$ cases of syphilis in pregnancy around the world. Of these, $39 \%$ were in Africa, $8 \%$ in the Americas, $44 \%$ in Asia and 1.6\% in Europe, and 1,085.637 had prenatal care. Untreated maternal syphilis resulted in 304,000 fetal and perinatal deaths, and more than 216,000 infected children at risk for early death. The majority $(87 \%)$ of these adverse events occurred in women who had prenatal care. In 2000, the WHO launched a campaign to eliminate syphilis MTCT with the intention to test and treat more than $90 \%$ of the pregnant women by 2015 . Unfortunately, this has not happened yet. ${ }^{3,6}$

The repercussions in the infected children's health can be severe. Syphilis occurrence during pregnancy can result in bad adverse effects for the concept , ranging from abortions, fetal or perinatal deaths, to newborns with diverse disease sequels that can be manifested up to 2 years of age. It is important to emphasize that $70 \%$ of the infected children have no symptoms at birth. Therefore, it is extremely important to screen and treat women during pregnancy. $2,4,7$

National guidelines determine screening for the infection twice during prenatal care: early in the pregnancy and at the beginning of the third trimester. Women should be tested again at admission to delivery (or to abortion). This strategy would identify women who had untreated infections prior to becoming pregnant and the ones who were infected during pregnancy, labor or delivery. The adequate identification would lead to the correct treatment, preventing fetal or neonatal infection. ${ }^{4}$

The great majority of the infected pregnant women are in the latent stage of the disease, when no disease symptoms are manifested yet. Hence, the importance of screening for the infection through laboratory exams, serological tests and rapid test. ${ }^{2,4}$

Identifying primary chancre of syphilis in pregnant women is not frequent since most of these lesions happen in the vagina or cervix and are not usually noticed by the patient. We must always consider the possibility of the presence of syphilis in any pregnant woman who mentions a previous or recent genital ulcer. The occurrence of a cutaneous rash found on the palms and soles must always bring up the possibility of secondary stage of syphilis. Another symptom characteristic of this secondary stage of the disease is the presence of flat condyloma with multiple surface papillae lesions, which can many times be misinterpreted as induced HPV lesions. The majority of women with diagnosis of syphilis during prenatal care are in the latent stage of the disease, which is characterized by reactive serological tests but no clinical manifestations or previous history of infection or treatment.

A study from 2006, which screened pregnant women for syphilis in the main cities in Brazil, found that $87 \%$ of them were in the latent stage. The risk for syphilis vertical transmission is high when the disease symptoms are present (primary and secondary stages), varying from 90 to $100 \%$. In the latent and tertiary stages, the risk varies from 10 to $30 \%$. Syphilis presents elevated rates of MTCT, and because Treponema is an agent with high molecular weight, its transmission will increase in the latest stages of pregnancy due to the augmented permeability of placental barrier along the pregnancy. Mother-to-child-transmission will be higher in the clinical manifestation stages of the disease also because the amount of circulating Treponema is larger than in the other stages. ${ }^{2,4}$

The strategies to identify the infection during pregnancy include laboratory tests since most of the women are asymptomatic. The blueprint screening diagram includes a non-treponemal test, which combines venereal disease research laboratory (VDRL) and Rapid Plasma Reagin (RPR) Test associated with treponemal tests including T Pallidum Hemagglutinin Assay (TPHA) or Fluorescent Treponemal Antibody Absorption (FTA-ABS). The non-treponemal test has the advantage of being highly sensitive, and its titers can help to evaluate the response to the treatment. Nevertheless, since they are based on the detection of anti-cardiolipin antibodies, these reactions can have false positive results. False reactive results occur in less than $2 \%$ of the screened uninfected individuals and they can be considered an exception to the rule. Another problem with the non-treponemal test is that false negative results can occur in up to $25 \%$ of the individuals in the latent stages of the disease. Since this situation is more frequently observed in pregnant women, it is recommended to screen for serum with non-treponemal and treponemal tests simultaneously. The treponemal tests are specific for syphilis and they confirm diagnosis. Nevertheless, even after successful treatment of syphilis, the tests still react and persist as a serum scar for the infection, and the patient can have positive results for treponemal tests forever. ${ }^{2,8}$

Another recently recommended strategy for syphilis screening is the so-called reverse algorithm strategy. It consists in initially having an automated treponemal test, which facilitates the testing of a large number of cases simultaneously. This screening uses immune enzymatic techniques (chemiluminescence immunoassay [CLIA] and chemiluminescent microparticle immunoassay [CMIA]) with the advantage of lower costs and automatization. Both present high sensitivity but lower specificity. When an immune enzymatic test is negative, one can discard infection, but when it is positive, confirmatory VDRL and TPHA/FTA-ABS tests are necessary. When the CLIA or CMIA is positive and VDRL and TPHA are negative, it is a case of false positive. When CLIA or CMIA is positive and VDRL and TPHA are positive, the syphilis infection is confirmed. These tests are recommended when there is well structured and efficient laboratory network. ${ }^{2,4,8}$

Since 2005, the Ministry of Health in Brazil recommends screening during prenatal care utilizing rapid test in situations where difficulties with laboratories network exist, or when there is an intention to expedite syphilis diagnosis for pregnant women. These tests have similar technology to the rapid tests for other infections, such as HIV. They are 
laboratory tests with excellent sensitivity and specificity. Because they are treponemal tests, when positive, they confirm the infection and indicate the treatment. They must be followed by VDRL, though, to evaluate responses to treatment since, even after treatment, the rapid test will not become negative, just like all the other treponemal tests (TPHA or FTA-ABS), which will not get negative after successful treatment ${ }^{4}$.

Syphilis treatment during pregnancy must use penicillin, since there is no evidence of any other drug that can treat the fetus intra-uterus adequately. The sooner the treatment initiates, the better for the fetus. Penicillin initiated after the 14th week is considered a treatment for a potentially infected fetus due to the high levels of intrauterine transmission of syphilis. Only first trimester treatment prevents fetal infection. ${ }^{9,10}$

The recommended penicillin doses are defined by the mother's diagnosis of recent or later stage of infection. When in the primary or secondary stages of the disease, that is, when there are clinical signs of the disease, the recommended dosage of penicillin regimen is $2.400 .000 \mathrm{UI}$ divided in two injections, one in each gluteus muscle. ${ }^{9,10}$

The majority of pregnant women will have no symptoms and no previous history of treatment or knowledge of the infection. In this situation, the diagnosis is undetermined latent stage of infection and the patient should be treated with 7.200.000 UI, divided in 3 weekly injections of $2.400 .000 \mathrm{UI}$.

The efficacy of penicillin in preventing and treating fetal infection is high. The most efficient treatment regimens are not yet established, although the ones in use are known to have highly successful rates. ${ }^{9,10}$

Another important point in syphilis treatment, as in any other sexually transmitted disease, is the adequate approach to treat the woman's sexual partner(s). Partners should be called to the health service to receive orientation, to be clinically evaluated, to collect serum and to be treated. If partners do not attend to the convocation, the prescription for medication should be sent through the pregnant woman, together with a written convocation from the health service. Partners, as well as patients, need to be screened for other STDs and to receive information about syphilis reinfection risks and ways to prevent it. Because of the cultural and social barriers associated with STDs, activities directed to partners need to be well structured. Health care workers need to be trained to learn how to deal with these situations efficiently. 2,4

Syphilis diagnosis in a pregnant woman requires adherence to an intensive follow up program, which should focus on preventing reinfection. Monthly VDRL testing is recommended after treatment, as well as the use of condom. Pregnant women should be alerted about the risks of any STD to the fetus, in special, syphilis infection. ${ }^{2,4}$
Adequate prenatal care should include preventive measures and treatment to avoid any risk to the fetus. Screening and treatment for syphilis are two actions that certainly have an impact in reducing morbidity and mortality rates among these children. We urgently need to sensitize health care professionals to routinely screen for syphilis during pregnancy, to correctly interpret the tests, and to treat these patients and their partners accordingly. If health professionals can be more aware of their role in preventing syphilis MTCT, and if they can incorporate adequate actions to their practice, we will certainly reach the same success we did before with preventing HIV infection transmission. Syphilis screening and treatment are way less expensive than those for HIV and should be available in every health care unit in our country. What might make an impact in preventing the spread of the infection is to adequately train the health care professionals in prenatal clinics to be aware of the syphilis problem and to act on it.

\section{References}

1 Ramos AN Jr, Matida LH, Saraceni V, Veras MA, Pontes RJ. Control of mother-to-child transmission of infectious diseases in Brazil: progress in HIV/AIDS and failure in congenital syphilis. Cad SaudePublica 2007;23(Suppl 3):S370-S378

2 Lago EG. Current perspectives on prevention of mother-to-child transmission of syphilis. Cureus 2016;8(3):e525

3 Arnesen L, Serruya S, Duran P. Gestational syphilis and stillbirth in the Americas: a systematic review and meta-analysis. Rev PanamSaludPublica 2015;37(6):422-429

4 Brasil. Ministério da Saúde. Secretaria de Vigilância em Saúde Departamento de DST, Aids e HepatitesVirais. Protocolo clínico e diretrizes terapêuticas para prevenção da transmissão vertical do HIV, sífilis e hepatites virais. Brasília (DF): Ministério da Saúde; 2015

5 Boletim Epidemiológico - Sífilis. Brasília (DF): Ministério da Saúde/Secretaria de Vigilância em Saúde/Departamento de DST, Aids e Hepatites Virais. 2015;4(1):

6 Newman L, Kamb M, Hawkes S, et al. Global estimates of syphilis in pregnancy and associated adverse outcomes: analysis of multinational antenatal surveillance data. PLoS Med 2013;10(2): e1001396

7 Gomez GB, Kamb ML, Newman LM, Mark J, Broutet N, Hawkes SJ. Untreated maternal syphilis and adverse outcomes of pregnancy: a systematic review and meta-analysis. Bull World Health Organ 2013;91(3):217-226

8 Swartzendruber A, Steiner RJ, Adler MR, Kamb ML, Newman LM. Introduction of rapid syphilis testing in antenatal care: A systematic review of the impact on HIV and syphilis testing uptake and coverage. Int J GynaecolObstet 2015;130(Suppl 1):S15-S21

9 Walker GJ. Antibióticos para sífilis diagnosticada durante el embarazo [Internet]. 2007 [citado 2015 Nov 14]. Disponible en: http://apps.who.int/rhl/reviews/CD001143sp.pdf

10 Clement ME, Okeke NL, Hicks CB. Treatment of syphilis: a systematic review. JAMA 2014;312(18):1905-1917 